\title{
Study on the Traffic Congestion Control Based on Big Data Rule Collecting
}

\author{
Bo Gao ${ }^{1}$ \\ ${ }^{1}$ Liaoning Police College, Dalian, Liaoning, 116036
}

Keywords: big data rule collecting; traffic congestion control; data and study

\begin{abstract}
With the rapid development of social economy and the acceleration of urbanization, the number of motor vehicles in the city has increased dramatically. The traffic congestion problem that Beijing faces at this stage is a problem that many international big cities have encountered during the development process. This is the economy. The overall performance of unbalanced social development, and the advent of the era of big data provide the conditions for scientifically governing urban traffic congestion.
\end{abstract}

\section{Introduction}

Big data rule mining is an important content of intelligent traffic cloud services. Only by predicting traffic flow in advance, especially predicting and analyzing the causes of traffic jams, can we more effectively support the functions of traffic guidance and service systems and improve intelligent transportation. System construction. In this paper, the big data mining method is used to study the pattern of traffic congestion spreading in the traffic network, establish an analysis model from the perspective of rule mining, establish a congestion warning prevention and control mechanism, and improve the unreasonable part of the road network construction plan, so as to improve traffic efficiency. , reduce the occurrence of congestion, assist in the study of the formation mechanism of traffic congestion, can serve both traffic management and demand management, and then have important significance for the governance of traffic congestion.

\section{Traffic Big Data}

Big data traffic congestion rule mining has important significance for intelligent traffic services. By establishing a time series traffic congestion conduction model and applying it to current traffic data, it is possible to predict changes in future traffic conditions and future traffic congestion. For example, drivers can learn about current traffic news through the traffic information system. However, this information is often received when the driver is already on the road and is on the road because of a traffic jam. If he can accurately predict the traffic conditions in the future, he or she will arrive before the congested road section. You can avoid the sections that will be congested in advance. Point out the road sections that are easy to congest, and then excavate the mechanism rules of traffic jams in the road network. Integrate these laws together and establish a time series of traffic congestion model. This model information can help the traffic management bureau and urban planners Good design, management and improvement of transportation systems. The congestion conduction model can also predict the traffic conditions and early warning of the occurrence of traffic jams, which is of great significance to the real-time navigation service technology of traffic. The model proposed in this paper can qualitatively and quantitatively describe the state of traffic, especially the transmission mechanism of traffic jams in urban transport networks, simulate and analyze the comprehensive effects of traffic congestion on the proliferation of traffic networks, and predict future traffic flow conditions to ease traffic. Congestion issues provide better theoretical guidance for designing, managing, and improving transportation systems. At the same time, this study can provide a new analysis paradigm for the study of transportation management:

1) The system platform of the model. The construction of the system analysis framework is an important basis and basis for research, which can provide guidance and analysis for research. Based on the theoretical research and practical investigation, this project will thoroughly explore the basic 
components of the big data rules mining platform for the study of the traffic congestion transmission mechanism. 2) Big data preprocessing subsystem --- preprocessing and data cleaning process of massive traffic flow data. The traffic flow data also has spatial-temporal correlation, and traffic flow data and other data are only meaningful when associated with a certain time and road. Intelligent traffic information mining of spatio-temporal rules has important application value for intelligent traffic cloud system prediction. How to embody the characteristics of spatio-temporal correlation in large data processing and database architecture, and perform two-dimensional data manipulation in time and space, is another focus of the data preprocessing subsystem. 3) Rules mining subsystem. Research on time series data mining algorithms for road traffic network can mine the time series association rules for effective use of mass traffic data, and store the mining association rules as rules for transmission in the rule base. 4) Rule Modeling Subsystem --Conduction Rule Modeling Research. The subsystem models the time-series association rule bases to form a time series summary for forecasting future traffic conditions, thereby establishing a traffic congestion conduction model and providing analysis support for the study of traffic congestion mechanisms. With the aid of data mining tools, systematically analyzes the conduction law and guidance model of traffic flow in traffic networks, analyzes the mechanisms of congestion formation, and applies them to traffic congestion analysis. 5) Traffic Analysis and Supporting Sub-modules - Traffic Flow Analysis and Policy Validation. It will be possible to study the congestion transmission mechanism and find out which road or road connection is more likely to conduct traffic congestion. What is the conduction path of traffic jams in the transportation network? What kind of management policies may ease traffic congestion? Providing this information to the traffic design and planning department will facilitate the scientific and rational design of traffic roads and help the traffic management bureau and city planners better design, manage and improve the transportation system.

\section{Characteristics of Traffic Congestion in Large Cities in China}

In China, with the continuous advancement of urbanization, the spatial concentration of economic production, population movement, and public services in large cities, combined with the restrictions on the supply of land resources, have caused urban traffic congestion to become a common problem faced by cities. Especially in big cities such as Beijing, Shanghai, Guangzhou and Shenzhen, the problem of traffic congestion is even more serious. The main road speed during peak hours is only $15-18 \mathrm{~km} / \mathrm{h}$. According to figures released by the Beijing Traffic Management Bureau, in 2014, the net increase in motor vehicles in Beijing was 154,000, the number of possessions increased by $2.8 \%$, and the roads in the newly built urban areas were 131 kilometers, an increase of only $2.1 \%$. In 2014, the city had 5.597 million motor vehicles, the total length of urban roads was 6426 kilometers, and 870 vehicles per kilometer were needed. Although the urban traffic congestion in our country is universal, due to the difference in the level of motorization, the urban traffic congestion in our country is diversified: (1) The traffic congestion is normal and the scope of congestion continues to expand; not only in large cities. Some second- and third-tier cities also face serious traffic congestion problems such as Jinan and Dalian. (2) Morning and evening peak tidal congestion. Congestion is mainly concentrated in main roads, and mainly in unidirectional congestion; (3) Congestion is prolonged during peak hours. The duration of traffic congestion on working days in Beijing (including severe congestion and moderate congestion) increased from 0.9 hours in 2009 to 1 hour and 55 minutes in 2014. (4) Major road network nodes and intersections are congested, constraining the efficiency of the entire road network, presenting a trend of congestion from "point" congestion to "point-line-surface" congestion.

\section{Causes and Countermeasures of Urban Traffic Congestion in China}

The most direct cause of traffic congestion is the imbalance between supply and demand. Taking Beijing as an example, the permanent population of Beijing has increased from 10.7 million in 2005 to 20.963 million in 2012. With the rapid growth of population, the demand for transportation has 
also increased dramatically. According to statistics, in 2012, the average daily travel volume in Beijing's Liuhuan reached 30.33 million (excluding walking), and the average number of trips at the end of 2011 was 28.73 million. In just one year, it increased 1.6 million people, an increase of 5.6\%. At the end of 2012, the total number of motor vehicles in the city was 5.2 million, a net increase of 216,000 units compared to the end of 2011 , an increase of $4.3 \%$. The speed of road construction is far lower than the growth rate of motor vehicles. From the data point of view, the number of motor vehicles in Beijing was 2.461 million in 2005, and the area of roads at the end of the year was 74.37 million square meters. In 2012, the number of motor vehicles in Beijing reached 5,200,000, an increase of $111.2 \%$ over 2005 . The annual road area should be 92.36 million square meters, an increase of only $24.2 \%$ over 2005 . This means that the density of motor vehicles on the unit road area has increased by 1.5 times compared with 2005.

The common problems in China's big cities are inadequate public transport investment, backward public infrastructure, and unclear public transportation in the urban transport system. Although the government has formulated a series of public transport development strategies, due to restrictions on fiscal expenditure, management, and road conditions, the status quo of urban public transport development is still not optimistic. Bus rights are not guaranteed. Although many large cities have set up bus lanes, special lanes do not form a network, which results in low speeds of bus traffic on congested roads. Moreover, the low rate of public transportation and low line density reduce urban public traffic. Attractive. In addition, the inconvenience of transfer between different modes of transportation is also one of the reasons for the low proportion of public transport. According to the results of the fifth comprehensive traffic survey in Beijing, a single subway trip takes 39\% of the total time outside the vehicle and 35\% of the ground-based public transport.

One of the reasons for urban traffic congestion is the rapid growth of cars, which leads to a relatively lagged traffic infrastructure. This leads to traffic congestion. Large cities such as London and New York have adopted a traffic demand management policy to maintain a dynamic balance of transportation supply and demand. The traffic demand management policy for small cars is an important part of urban transport policies, and they are purchased in cars through economic, administrative, publicity and education. Relevant policies have been formulated on the links of card, pass, and parking to control the growth of vehicles in an orderly manner and guide the rational use of cars. Improving the purchase threshold of cars and the cost of car use can, to a certain extent, guide residents to change their mode of travel and reduce the pressure on the transportation system.

The type of land use is closely related to the transportation system. The two promote each other and restrict each other. The types of land use, development intensity, and spatial connections will affect the residents' travel distance, travel time, and choice of travel mode to some extent. On the contrary, the transportation system determines the spatial accessibility of land use and further influences the type and value of land utilization. It is difficult to effectively solve the congestion problem by simply increasing the supply of transportation capacity, and it is necessary to coordinate the management of transportation demand, land use, and transportation layout. Among them, the traffic-oriented development model is the main direction. Large cities such as Singapore, Hong Kong, and Tokyo all attach great importance to the guiding role of transportation in land use. To avoid increasing commuting distance, urban construction and expansion are mainly carried out along urban rail transit lines. For many domestic cities that are also faced with the problem of scarcity of land resources, it is of great significance for reference.

Vigorously developing public transportation is the basic guarantee for increasing the threshold for buying cars and guiding residents to change their mode of travel. After the 1950s, Tokyo began to build rail transit on a large scale to solve the problem of traffic congestion in the city. Cities like Seoul and Hong Kong have also taken the same measures. In the 1990s, due to the lag in transportation infrastructure construction in Seoul, South Korea, the problem of urban traffic congestion was serious. To ease traffic congestion, Seoul has accelerated the construction of urban rail transit. It also began to actively promote reforms in public transport, build a central bus lane, reintegrate lines, introduce GPS devices, and use new parking management systems. After the reform of public transport, the number of public transport (bus and subway) passengers increased 
by $11 \%$, while fuel consumption and air pollution gradually decreased, and bus-related traffic accidents decreased by $26.9 \%$.

Only by increasing the supply of transportation can not fundamentally solve the problem of traffic congestion. Civilized travel awareness and good travel habits are the key to solving traffic problems. In recent years, foreign cities have put forward the idea of building green cities and livable cities, and encourage citizens to choose green travel modes. Copenhagen is a typical city for green travel. It has been selected by UN-HABITAT as the "best place to live in." More than $40 \%$ of citizens choose to ride a bicycle to work, so Copenhagen has become a famous bicycle city. To encourage people to travel green, London has established a number of bicycle rental points in the urban area. It has a total of approximately 10,000 bicycles and is open all day long.

\section{Conclusion}

From a longer-term perspective, we should promote the integration of traffic data collection, traffic operation monitoring, traffic congestion forecasting and simulation, traffic demand induction and mitigation congestion policy implementation and effect forecasting, implement modularization, platformization, strategy, intelligence The management of congestion policy and the construction of a smart city system, and the establishment of a closed-loop traffic monitoring and demand management system platform for "module application-analysis-feedback-congestion assessment—prediction—selection strategy—module application”.

\section{References}

[1] Wang Kai-Ying, Yan Bingqiu, Wang Fang, et al. Foreign plans for prevention and treatment of urban diseases and their ideas and measures for reference [J]. World Geography, 2014(01):65-72.

[2] National Bureau of Statistics. China Statistical Yearbook 1995 [J]. 1995: 1-20.

[3] Zhao PJ, Li Y. Theoretical analysis of the role of big data method in alleviating urban traffic congestion [J]. Modern Urban Research, 2014(10): 25-30.

[4] Guo Jifu, Liu Ying, Yu Liu. Understanding of the problem of traffic congestion in China's big cities [J]. Urban Transport, 2011(02): 8-14.

[5] Lin Xiongbin, Yang Jiawen. Multi-dimensional Comprehensive Review of Urban Traffic Congestion Characteristics and Governance Strategies [J]. Comprehensive Transportation, 2015 (08): 55-61. 